\title{
Horizontally oriented v-y flap may diminish lower lid retraction
}

\section{Javad Rahmati 1, 2, Ali Atri ${ }^{1}$, Shahriar Haddady Abianeh 1, 2, Hojjat Molaei 1,3*}

${ }^{1}$ plastic \& reconstructive surgery department, Vali-e-Asr Hospital, IKHC, Medicine School, Tehran University of Medical Sciences, Tehran, Iran ${ }^{2}$ plastic \& reconstructive surgery department, Razi Hospital, Medicine School, Tehran University of Medical Sciences, Tehran, Iran ${ }^{3}$ plastic \& reconstructive surgery department, Sina Hospital, Medicine School, Tehran University of Medical Sciences, Tehran, Iran

*corresponding Author: Hojjat Molaei, Vali-e- Asr Hospital, Imam Khomeini Hospital Complex, Bagherkhan St., Towhid Sq., Tehran , Iran. Received Date: August 28, 2020; Accepted Date: September 07, 2020; Published Date: September $10,2020$.

Citation: Rahmati J, Atri A, Shahriar H Abianeh, Molaei H., (2020) Horizontally oriented v-y flap may diminish lower lid retraction. Journal of Surgical Case Reports and Images, 3(4); Doi: 10.31579/2690-1897/034

Copyright: () 2020 Hojjat Molaei, This is an open-access article distributed under the terms of the Creative Commons Attribution License, which permits unrestricted use, distribution, and reproduction in any medium, provided the original author and source are credited.

\section{Abstract}

Introduction: lower lid malposition following malignancy surgery in infra- orbital area is often challenging and any way to reduce its chance is appreciated.

Case presentation: 36 years old women with confirmed Basal Cell Carcinoma (BCC) of left cheek underwent tumor excision with free margins and remained skin defect reconstructed with adipocutaneous v-y advancement flap, which designed in a horizontally orientation. Patient had no signs of ectropion with good wound repair and obscured scar.

Conclusion: v-y flaps are commonly used local flaps with minimal donor site morbidity, and if designed horizontally, not only may reduce chance of lower lid malposition, but also can remain more acceptable scars.

Key Words: v-y flaps; facial oncologic; tumor margins

\section{Introduction}

Recently enhanced reconstructive techniques have greatly improved facial oncologic reconstruction. Concerns about tumor margins, probable radiotherapy, and lymph node dissection makes surgeons to have different plans to overcome complications. Cheek area as one of attractive and aesthetic facial elements, is predisposed to cutaneous malignancies. There are optional solutions to cover the resultant skin defect, which demands selective technique according to final pathology and free margins. [1]

Lid malposition is a difficult complication after lower lid procedures, lidcheek junction, and even cheek surgeries. This malposition can be due to excess skin resection, scarring, or improper tension of the tarsoligamentous slings. In untreated cases, this can result in exposure keratopathy, which is harmful, especially in careless clients. [2]

Among recommended procedures to reconstruct a skin defect after tumor excision, $\mathrm{V}-\mathrm{Y}$ flap is usually aesthetically superior to full-thickness skin grafts, but the pincushion effect is very common and often requires a second surgery to reshape the nasofacial sulcus [3].

$\mathrm{V}$-y flaps as workhorse instruments in reconstructive surgeons` hands have their advantages, such as preserved sensation, minimal donor site morbidity, and various spatially oriented, and better learning curve [4]. So selection of proper direction of incisions is applicable according to Langer lines of skin.

Our previous experience had demonstrated superiority of this flap in avoiding lower lid retraction in malar area reconstructions, which can guide surgeons as safe technique, and here we present a similar case.

\section{Case presentation}

A 33 years old female was consulted for her cheek lesion for a 5 months from dermatologist. She had tried some topical treatments but persistent progressive lesion in her cheek forced her to look for another therapies. Tissue examination biopsy of lesion reported as Basal Cell Carcinoma (BCC), nodular subtype.

When she referred to us, she had a flat no ulcerated solitary lesion in left medial cheek near nasolabial fold, without any pain or itching (figure 1).

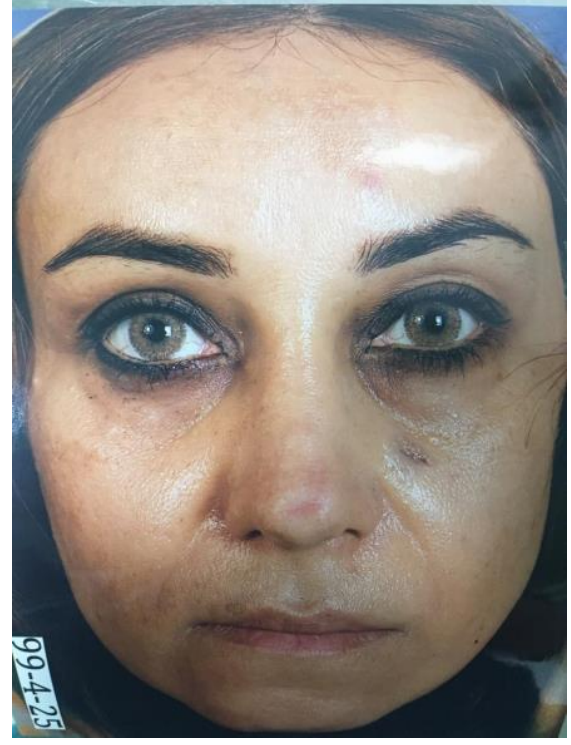

Figure 1: 33 years old female with facial left side BCC 
She was prepared to cancer surgery and lesion was removed with $5 \mathrm{~mm}$ safe margin (confirmed by tissue examination). So, there was a considerable skin defect near lid- cheek junction (figure 2).

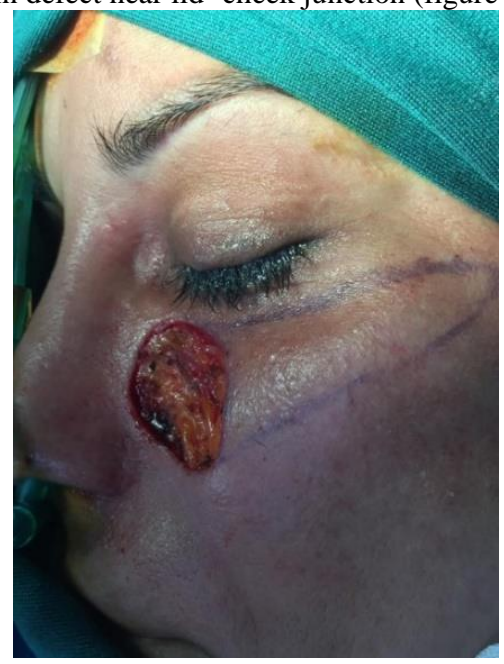

Figure 2: Skin defect after tumor excision with free margins.

A horizontally oriented $v-y$ flap with apex in lateral facial area was designed as illustrated in figure 2 . The surgery promoted as routine adipocutaneous v-y flap, and the defect covered without ant traction or extra tensions. She came back several weeks with good and acceptable scar and satisfied with her resultant face and relaxed lower lid without retraction (figure 3 ).

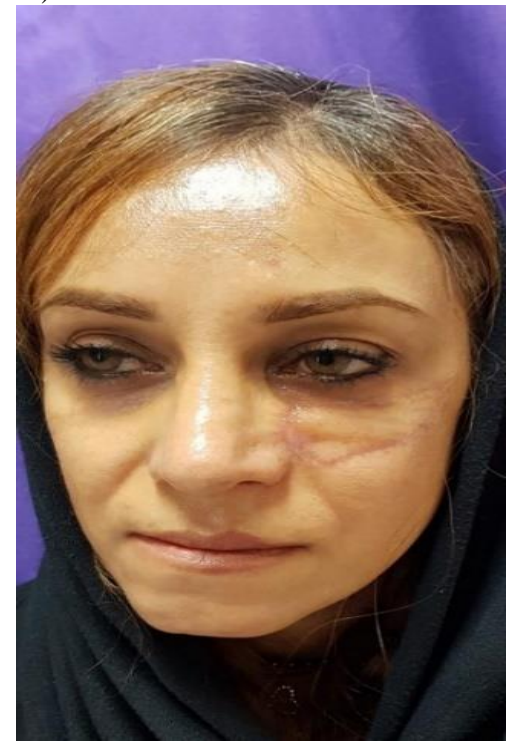

Figure 3: Same patient weeks later with intact lower lid position

\section{Discussion}

Nowadays, oncoplastic surgery should answer some key questions about complete tumor eradication, suitable defect coverage and less donor site morbidity. Facial malignancies may be assumed challenges between surgeons to reach improved results. Flaps are reconstructive surgeries superior to grafts to cover skin defects.
V-Y flap, as an island-like skin with subcutaneous tissue connected to it, has been widely employed in different styles [4]. Limited rotation or deformation remains smooth incisions which usually is similar to triangular kite - kite flap- to reach the real distribution of tissue stress [5].

Dölen et al (2018) designed templates to V-Y rotation advancement flaps as unilaterally or bilaterally to have the lest donor site pincushion and morbidity. These templates were designed according to defect diameter in which, the length of a unilateral was equal to 3 times the horizontal radius ( $\mathrm{r}$ ) of the defect $(\mathrm{BC}=3 \mathrm{r})$. The length of the bilateral $\mathrm{V}-\mathrm{Y}$ rotation advancement flap was equal to twice the radius $(B C=2 r)[6]$. Their plan enhanced quality of donor site closure without compromising viability of flap. But, they did not mention the importance of flap orientation as we considered.

Sugg et al (2013) demonstrated that inferior-to-superior V-Y advancement flap for the reconstruction of lid-cheek junction defects was similar to the Mustardé flap for ectropion prevention. As. We know, weight of larger flaps in lower lid area reconstructions, may predispose to future malpositions and complications unless supports. They used tension redistribution techniques in stair-step pattern of deeper layers and prepare an area of equally distributed tension that eventually prevented ectropion [7].

We present here our preference to choose v-y flap in infra-orbital area in a horizontally orientation to diminish chance of future ectropion of lower lid, which is usually difficult to manage. Our patients were overall satisfied and they did not experienced ectropion in follow up visits. On the other hand, it seems final scars would be more acceptable, and we can recommend surgeons to consider such minor modifications during flap marking and design.

Conflict of interest: Authors have not any conflict of interest.

\section{References}

1. Heller L, Cole P, Kaufman Y. (2008). Cheek reconstruction: current concepts in managing facial soft tissue loss. Semin Plast Surg. 22(4):294-305.

2. Alghoul MS, Kearney AM, Pacella S J, Purnell, C A. (2019). Eyelid Reconstruction. Plastic and Reconstructive Surgery Global Open, 7, 11 - p e2520.

3. Rossi M, Milia A, Carmisciano M, D'Arpa S, Cordova A, Moschella F. (2013). Advancement perforator cheek flap for aesthetic one-stage reconstruction of postoncological extended split-thickness defects of the nasal sidewall. ScientificWorldJournal. 169208.

4. Thoma A, Vartija LK. (2010). Making the V-Y advancement flap safer in fingertip amputations. Can J Plast Surg, 18(4):e47e49.

5. Yang ZL, Peng YH, Yang C, Cheng B, Ji MK, Zhao Y. (2020). Preoperative Evaluation of V-Y Flap Design Based on Computer-Aided Analysis. Computational and Mathematical Methods in Medicine, Article ID 8723571, 9.

6. Dölen UC, Koçer U. (2018). Innovation in the planning of VY rotation advancement flaps: A template for flap design. Arch Plast Surg, 45:85-88

7. Sugg KB, Cederna PS, Brown DL. (2013). The V-Y advancement flap is equivalent to the Mustardé flap for ectropion prevention in the reconstruction of moderate-size lidcheek junction defects. Plast Reconstr Surg, 131(1):28e-36e. 